\title{
Effects of amount of attention allocated to the location of visual stimulus pairs on perception of simultaneity
}

\author{
RODNEY A. CARVER and VINCENT BROWN \\ University of Texas, Arlington, Texas
}

\begin{abstract}
This study examined whether a change in the amount of attention equally allocated to two locations affects judgments of the simultaneity or successiveness of stimuli presented at those locations. Observers were cued to expect two brief flashes either to the left and right of fixation or above and below fixation. Stimulus onset asynchrony was randomly varied. On a small proportion of trials, the stimuli appeared at the unexpected locations. Observers were more likely to report the stimuli as simultaneous when they appeared in the unexpected locations. A model proposed to account for the data assumes that a brief stimulus event is represented by a probability distribution reflecting the uncertainty in determining the time of the event's occurrence, and two events are judged to be simultaneous if they are perceived to fall within some critical temporal interval, $c$, which is a function of the amount of attention allocated to the task.
\end{abstract}

It has long been recognized that the perceived order of a pair of closely timed stimuli does not always correspond to the actual order of their occurrence (see James, 1890). The idea that some stimuli could receive perceptual or attentional priority for further processing led to the notion of "prior entry," which was the notion that there were factors that could alter the time it took for a stimulus to reach awareness (see Boring, 1950). The physical intensity of a stimulus is one strong determinant of prior entry (e.g., Roufs, 1963). More recently, Sekuler, Tynan, and Levinson (1973) uncovered what seemed to be an inherent bias in the visual modality toward more accurate recognition of the temporal order of left-first-right-second stimuli over right-first-left-second stimuli. The observed bias could be due to a left-right scanning mechanism such as is assumed to occur in reading, which would make the bias an attentional effect. Using a location-cuing manipulation, Stelmach and Herdman (1991) demonstrated that the direction of attention could bias the perception of the temporal order of two visual stimuli: stimuli appearing in the attended location were more likely to be perceived first than were stimuli appearing in the unattended location (see also Hikosaka, Miyauchi, \& Shimojo, 1993).

While it is clear that an attentional bias can affect judgments of temporal order and simultaneity, a related question that, to our knowledge, has not yet been addressed is whether the overall level of attention directed toward both stimuli in a temporal order task has any effect on judgments of temporal order or simultaneity. Does attention increase the precision of judgments of the relative time of

The authors wish to thank Arthur Kramer and two anonymous reviewers for their helpful comments on earlier versions of this paper. Correspondence can be addressed to either R. A. Carver or V. Brown, Department of Psychology, University of Texas, Arlington, TX 760190528 (e-mail: rcarver@uta.edu or brown@uta.edu). occurrence of two stimulus events? Conversely, to what extent are such judgments harmed by a decrease in the amount of attention focused on the task?

A number of models of temporal order perception have been proposed, and, as Sternberg and Knoll (1973) point out in their review, the proposed models are all forms of a general independent-channels model. In this very general model, the sensory channels stimulated by the events of interest are assumed to be independent in the sense that the activity in one channel does not influence that in another, in terms of either what occurs or when it occurs. Because the internal transmission and detection times are assumed to be probabilistic, the difference in arrival times at the decision mechanism for two stimuli will, in general, not be the same as the difference in the initial stimulus times. Several new models and refinements of older ones have been proposed since Sternberg and Knoll's review (e.g., Allan, 1975; Jaśkowski, 1991; Stelmach \& Herdman, 1991; Ulrich, 1987), but none can be said to deviate from the general independent-channels foundation.

Allan (1975) used a two-decision paradigm in which observers were asked on each trial first to render a judgment of successiveness or simultaneity regarding the relative offsets of a tone and a light and subsequently to make a forced-choice order decision. Allan, who first examined a perceptual latency model which postulates a variable lag between the occurrence and the perception of stimuli, assumes that the perception of successiveness and the perception of order are determined from the same internal event. Her data were inconsistent with this model. In place of the rejected perceptual latency model, she proposed an attention-switching model that was consistent with her data. She proposed that the time at which a stimulus was judged to occur depended on when attention was switched to the relevant stimulus channel; thus, if one were attending to one channel when a stimulus arrived 
on another channel, the judged arrival time of that stimulus would be delayed.

Ulrich (1987) examined the predictions of Allan's (1975) attention-switching model along with two other particular cases of what he called the general threshold model (triggered-moment and perceptual-moment models). The general threshold model also assumes that the perception of successiveness and the perception of order are determined from the same internal event. Ulrich used a ternary response-category approach in which the three available responses were "stimulus $x$ before $y$ " (" $x y$ "), "stimulus $y$ before $x$ " (" $y x$ "), and "simultaneous" ("si"). On the basis of the shapes of the psychophysical functions resulting from observers' judgments of two visual stimuli, Ulrich rejected all but a modified attention-switching model in which the time spent attending to a channel after switching attention to that channel decreased with stimulus intensity.

Jaśkowski (1991), building on Ulrich's general threshold model, proposed a two-stage model in which there were two evaluators involved in the order-discrimination task: one that makes a simultaneous versus successive discrimination and one that makes a determination of order. The observer responds after the states of the evaluators are registered. The simultaneity evaluator is checked first, and if "si" is registered (when the difference in arrival times of the two stimuli is less than the simultaneity threshold, $\left.\left|A_{s x}-A_{s v}\right|<c_{\mathrm{s}}\right)$, a response is made accordingly. Otherwise, the order evaluator is checked, and if " $x y$ " or " $y x$ " is registered (when the signed difference in arrival times of the two stimuli exceeds the order threshold, $A_{\mathrm{o} x}-A_{\mathrm{o} y}>c_{\mathrm{o}}$ or $A_{\mathrm{o} y}-A_{\mathrm{o} x}>$ $c_{0}$ ), the corresponding order response is made. If, however "?" is registered in the order evaluator $\left(\left|A_{\mathrm{ox}}-A_{\mathrm{ov}}\right|<\right.$ $c_{\mathrm{o}}$ ), then there is no order information available and the response is made at random from among the three available response categories. The important difference between Jaśkowski's (1991) two-stage model and the approaches of Allan (1975) and Ulrich (1987) is that it is no longer assumed that the perception of successiveness and the perception of order result from the same internal event: both the arrival times and the thresholds are allowed to differ for the simultaneity comparator (which determines the perception of successiveness) and the order comparator. Using the predictions of his model, Jaśkowski (1991) derives psychometric functions to which he fits Allan's (1975) bimodal (light and tone) data and Ulrich's (1987) unimodal (two lights) data with remarkable accuracy.

One common feature of the above models is that they make no direct allowance for effortful allocation or overt bias of attention; that is, there is no role for expectant attention. Allan's (1975) and Ulrich's (1987) attentionswitching models postulate that one of the relevant channels is attended at any given time, but without a mechanism for controlling with what intensity (effortful allocation) attention is given.

Stelmach and Herdman's (1991) model of temporal order perception explicitly incorporates expectant atten- tion. Their model is similar to Jaśkowski's (1991) model in that it incorporates the same two discrimination evaluators (simultaneity and order), but with the added element of specifying the nature of the input to and output from the two evaluators. The input to the evaluators is modeled after the Roufs and Blommaert (1981) temporal impulse response function: ${ }^{1}$

$$
k\left(\frac{t}{b}\right)^{3} e^{-\left(\frac{t}{b}\right)}
$$

where the parameter $t$ is time in milliseconds, $b$ is a free parameter which controls the growth and decay rates of the curve, and $k$ is a normalizing constant, $(e / 3)^{3}$, ensuring that the function's peak value is 1 . The function is unimodal and positively skewed, taking on values from 0 to 1 for positive values of $t$. This function is used by Roufs and Blommaert (1981) to model transient responses of the visual system to fast luminance variations (flashes) occurring within a foveal field, and thus appears reasonable to use in the present context.

A response represented by this function is assumed to be generated in each channel stimulated by the events of interest. In the case of two successive events, the onsets of the impulse responses to the two events are separated by an amount of time related to the difference in the onsets of the two events. The simultaneity evaluator then computes a ratio of common-to-uncommon areas under the two input curves, and the order evaluator computes the difference between the two inputs. The likelihood of a pair of events being judged as occurring simultaneously is thus directly related to the ratio of common-to-uncommon areas beneath the curves. The idea of using the ratio of common-to-uncommon areas comes from the work of Groner, Bischof, and DiLollo (1989).

Stelmach and Herdman (1991) propose that the value of the parameter $b$ is indicative of the amount of attention devoted to the events of interest, with greater attention decreasing the magnitude of $b$ and thus narrowing the impulse response function by increasing its rate of rise and decline. This would explain how attending more strongly to one stimulus than to another might cause a perception of successiveness when, in fact, the stimuli are simultaneous, since the impulse response to an attended event would peak more quickly than the impulse response to an unattended event. Reversals in the perceived temporal order of stimuli are accounted for in a similar fashion. ${ }^{2}$

In addition, although Stelmach and Herdman (1991) do not emphasize it, their model also predicts that two stimuli that are equally attended (perhaps occurring in locations to which attention has been directed by preceding cues) should generate inputs to the evaluators represented by equally small values of $b$, whereas two stimuli that are equally unattended (perhaps occurring in locations away from attentional cues) should generate inputs to the evaluators with commensurately larger values of $b$. For a given stimulus onset asynchrony (SOA), smaller values of $b$ (representing attended events) will generate temporal impulse response functions that are narrower, and 
thus have a smaller proportion of their areas in common than do the wider curves generated by larger values of $b$ (representing unattended events). These two classes of events (equally attended and equally unattended) should result in, respectively, lesser and greater likelihoods of registering " $\mathrm{si}$ " in the simultaneity evaluator due to the inverse relationship between the allocation of attention and the width of the resulting temporal impulse response functions. Figure 1 plots the ratio of common-to-uncommon areas for two successive copies of Equation 1 as a function of SOA for three values of $b .^{3}$

Assuming a direct relationship between the ratio of common-to-uncommon areas and a judgment of simultaneity (as do Stelmach \& Herdman, 1991), Figure 1 clearly shows that the proportion of such judgments will vary as a function of the attention parameter $b$. Higher values of $b$ give rise to a greater proportion of judgments of simultaneity at all SOAs. In other words, the amount of attention allocated to the stimulus locations should have an effect on judgments of the relative time of occurrence of the stimuli. Note that a straightforward interpretation of the notion of prior entry, which assumes that attention simply increases the internal transmission rate of stimulus information, would not predict different response curves for different levels of attention to both elements of a stimulus pair, since the speeds of transmission of information regarding both stimuli would be changed by the same amount.

If, then, we require a response from observers only when two target events are perceived as simultaneous as opposed to successive, we should expect more "simultaneous" judgments when attention is directed to two locations on an axis perpendicular to the event axis than when attention is directed to the locations of the target events themselves. These results would indicate that the overall amount of attentional resources devoted to the task can affect judgments of the relative time of occurrence of two equally attended stimuli.

In the experiment presented here, the target pair of events was presented on either of two axes (to each side of a center fixation dot or above and below the dot) with equal probability. Prior to the appearance of the target stimuli, a signal appeared which indicated the axis along which the stimuli should be expected. The cue indicated the correct stimulus axis $80 \%$ of the time and indicated the axis orthogonal to the stimulus axis $20 \%$ of the time. It was hypothesized that the number of responses indicating that the observer perceived the events as simultaneous would be greater in the incorrectly cued condition than in the correctly cued condition.

As a check on the effectiveness of the cue in directing attention to the intended axis on a given trial, 27 naive untrained observers participated in response-time trials in which only one stimulus occurred in any one of the four randomly chosen locations. The observers were told that the stimulus would occur in one of the two cued locations most of the time. The cues and the sequence of displays used in these trials were identical to those used in the main experiment (see Figure 2), and the single stimulus occurred in one of the cued locations $80 \%$ of the time. The observers were asked to respond with a keypress as quickly as possible when the stimulus was detected. Response times were faster in the valid-cue condition $(M=380 \mathrm{msec})$ than in the invalid-cue condition $(M=403 \mathrm{msec}$ ), and a 4 (location) $\times 2$ (cue validity) repeated measures analysis of variance supported the hy-

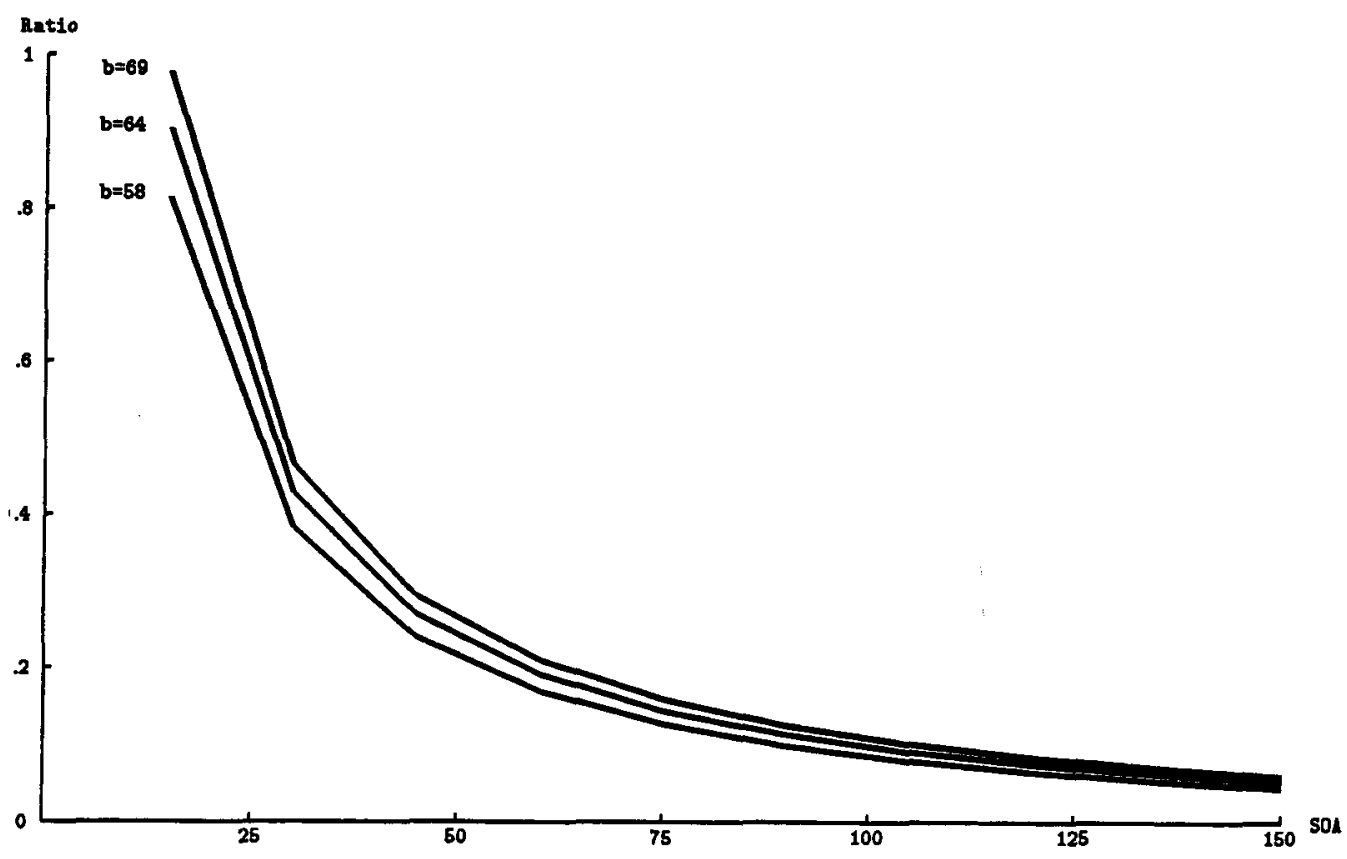

Figure 1. Output of the simultaneity comparator for different values of $b$ from the model of Stelmach and Herdman (1991). 


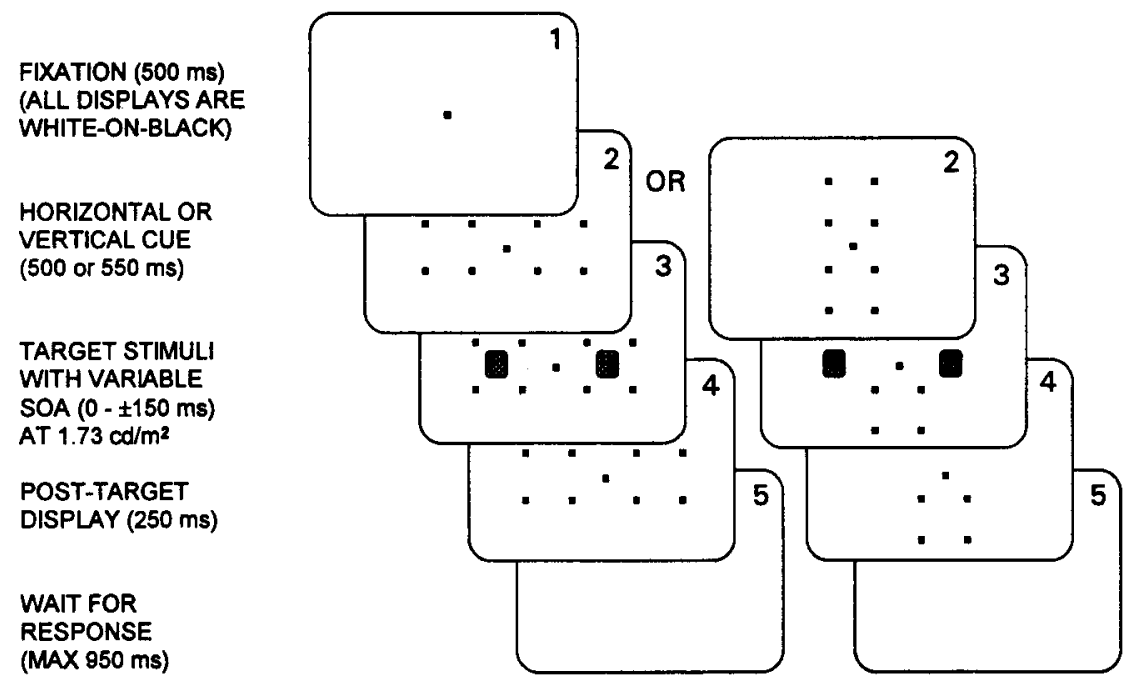

Figure 2. Sequence of stimuli. The valid cue (left panels) and invalid cue (right panels) for the horizontal targets are shown.

pothesis of a main effect of cue validity $[F(1,26)=34.7$, $p<.01]$. Neither the effect of cue location nor the interaction of location $\times$ cue validity were significant in the analysis $(F<1)$.

\section{METHOD}

\section{Observers}

The observers were 8 volunteers from among faculty and from among graduate and upper level undergraduate students at the University of Texas at Arlington who had duties in the laboratory where this research was being done. Two of the observers were the authors.

\section{Apparatus and Stimuli}

The stimuli were presented on a 15-in. NEC Multi-Sync 4FG SVGA color monitor controlled by an IBM-compatible 486DX computer. The vertical refresh rate of the monitor was set to $60 \mathrm{~Hz}$. The monitor was RGB balanced by observation to a neutral white, and calibrated using a Photo Research Spectra photometer to laboratory standard luminance and contrast such that the luminance of the light gray (LG) was approximately $12.0 \mathrm{~cd} / \mathrm{m}^{2}$. The grays used to produce the stimuli were those included in the standard set of $16 \mathrm{col}-$ ors provided by the computer's operating system.

A single LG pixel in the approximate center of the black display screen served as the fixation point. Target stimuli were 4 pixel $X$ 4 pixel (approximately $1.7 \times 2.1 \mathrm{~mm}$ ) rectangles, subtending approximately $0.20^{\circ}$ of visual angle horizontally and $0.24^{\circ}$ vertically at a viewing distance of $50 \mathrm{~cm}$. The luminance of each of the target stimuli was approximately $1.70 \mathrm{~cd} / \mathrm{m}^{2}$, the dark gray (DG) from the standard 16 available colors. These stimuli were set against a black background (luminance $<0.02 \mathrm{~cd} / \mathrm{m}^{2}$ ). The experiment was performed in near total darkness.

The target stimuli appeared in pairs on either side of the fixation point and were separated in time by an SOA chosen randomly with equal probability from intervals ranging from 0 to $\pm 150 \mathrm{msec}$ in increments of $16.7 \mathrm{msec}$. A negative SOA indicates that the left stimulus appeared first; a positive SOA indicates that the right stimulus appeared first. At a viewing distance of $50 \mathrm{~cm}$, the array of stimulus positions subtended a visual angle of approximately $3.8^{\circ}$ horizontally or approximately $2.5^{\circ}$ vertically, center to center.

\section{Design and Procedure}

The method used to direct attention to the axis on which a given target stimulus pair would appear is illustrated in Figure 2. Four corner pixels (luminance LG) defined cuing boxes that were presented along either the vertical or horizontal axis on every trial. These attentional cues were selected at random from both possibilities (vertical and horizontal) with equal probability. On $80 \%$ of the trials, the cue correctly designated the axis on which the target events would take place, and on $20 \%$ of the trials it incorrectly designated the axis orthogonal to the event axis.

The sequence of displays on each trial was as follows: (1) A single pixel warning dot centered between the boxes cuing the stimulus locations was presented for a duration of $500 \mathrm{msec}$. (2) The cuing boxes then appeared to either side (left/right or above/ below) of the warning dot for a randomly chosen interval of either 500 or 550 msec. (3) The two target stimuli were presented with a randomly chosen SOA between -150 and $150 \mathrm{msec}$ along either the vertical or horizontal axis for a duration $16.7 \mathrm{msec}$ each. (4) The boxes and focus dot remained on for $250 \mathrm{msec}$ following the offset of the second target stimulus. (5) An additional interval of up to $950 \mathrm{msec}$ of black screen was allowed for a response (observers could respond any time following the onset of the first target stimulus). The intertrial interval was $300 \mathrm{msec}$. Observers were instructed to press the right-hand shift key when the two target events were perceived as simultaneous. The dependent variable was the proportion of keypresses for each SOA in the two cue conditions (validly vs. invalidly cued) for both the vertically and horizontally oriented targets.

The sessions were preceded by a $10-\mathrm{min}$ period of dark adaptation in the darkened room where the experiment was conducted, during which 40 practice trials were performed. At the end of the 10-min dark adaptation, the test block of 380 trials was performed; included was a rest period (the length of which was determined by each individual observer) after every 64 trials. Five of the 8 observers completed 17 to 20 sessions of 380 trials each. The other 3 observers completed 9 to 11 sessions each.

\section{RESULTS}

The overall proportion of "simultaneous" responses collapsed across observers as a function of SOA and cue 
validity for both vertical and horizontal target orientations is shown in Figures 3 and 4.

As can be seen in the figures, the hypothesis that the proportion of simultaneous judgments would be greater in the invalid-cue condition was confirmed. Although the differences are not large, they are present at each of the individual SOAs, with the exceptions of those at the very tails of the response distributions. A three-factor (cue validity $\times$ SOA $\times$ target orientation) repeated measures analysis of variance of the proportion of "simultaneous" responses from all observers, collapsed across sessions, confirmed that the differences between the valid-and invalid-cue conditions apparent on the graphs were statistically significant $[F(1,7)=10.9, p<.02]$. (A chi-square test of individual observers' responses verified that the effect of cue validity was significant $-p<.01-$ for 6 of the 8 observers.) The effect of SOA was highly significant $[F(18,126)=94.1, p<.01]$. The effect of target orientation (horizontal vs. vertical) was not significant $(F<1)$. However, the two-way interaction between cue validity and target orientation was marginally significant $[F(1,7)=5.2, p<.06]$, indicating that cue validity had a greater effect on the vertically oriented targets than on the horizontally oriented targets.

The observers were more likely to report the stimuli as being simultaneous when they appeared at the uncued locations. This indicates that the amount of attention allocated to the stimulus locations affects observers' ability to make judgments of the simultaneity of stimuli appearing at those locations. Decreased attention would seem to decrease the precision of simultaneity judgments.

\section{DISCUSSION}

The experiment presented here indicates that observers are less precise in judging the simultaneity of two visual stimuli when attention is directed away from the locations of the stimuli.

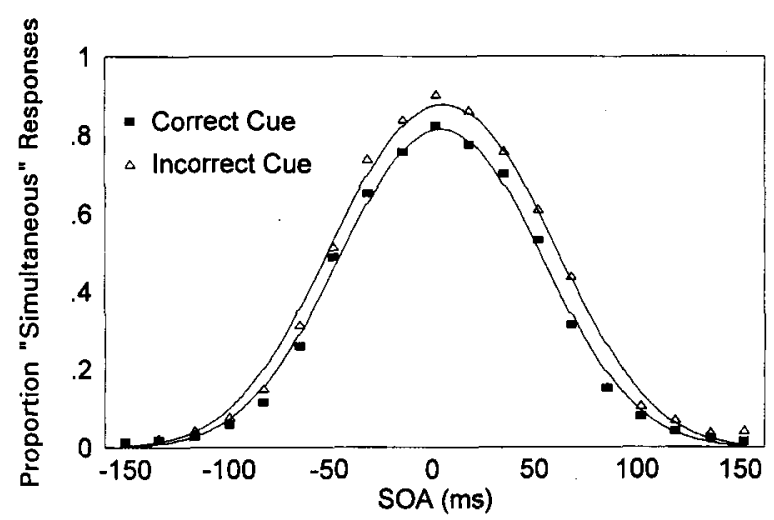

Figure 3. Distribution of "simultaneous" responses to vertical targets as a function of SOA for the valid- and invalid-cue conditions. The curves drawn through the data points represent the curves determined by the best fitting parameters for the model described in the Discussion.

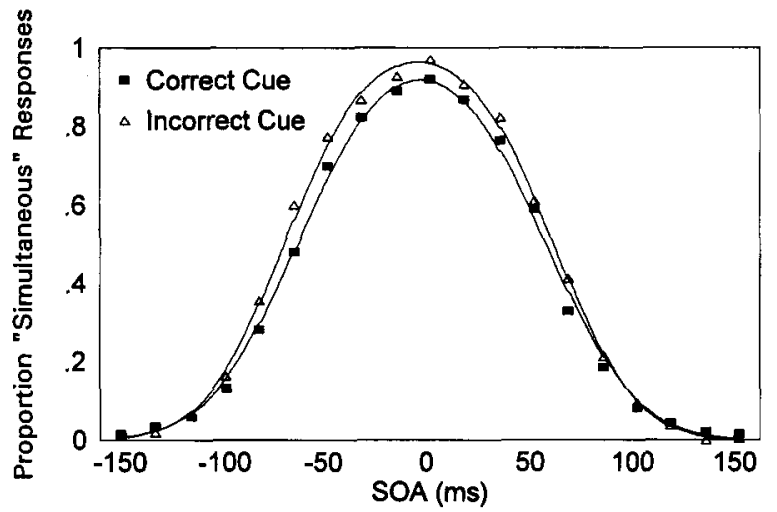

Figure 4. Distribution of "simultaneous" responses to horizontal targets as a function of SOA for the valid- and invalid-cue conditions. The curves drawn through the data points represent the curves determined by the best fitting parameters for the model described in the Discussion.

There are three points to notice about the empirical response functions for attended versus unattended stimuli: (1) The proportion of simultaneous responses is always less than 1 , even at zero SOA; (2) the valid-cue ("attended") response curve lies completely below the invalidcue ("unattended") curve; and (3) the curves appear roughly Gaussian in shape (i.e., they are unimodal with inflection points at intermediate SOAs).

Although the model proposed by Stelmach and Herdman (1991) is in qualitative agreement with both the results reported here and the results of their own experiments, it turns out that the precise form of the obtained SOA response function (see Figures 3 and 4 and Stelmach \& Herdman, 1991) is different from that strictly predicted by their model, using the ratio of common-to-uncommon areas under the impulse-response curves to determine judgments of simultaneity. When the impulse-response ratio is assumed, as do Stelmach and Herdman (1991), to directly determine observers' simultaneity judgments, the impulse-response model predicts a response function that is convex on each side of a center peak (see Figure 1). The data clearly show a Gaussian-like response function.

It is possible that there are modifications of Stelmach and Herdman's (1991) model which could generate the desired response curves. For example: other forms of the impulse-response function could be assumed; rules for determining stimulus simultaneity other than the ratio of common-to-uncommon areas of the impulse responses could be hypothesized; and, lastly, an appropriate mapping of the output of the hypothetical simultaneity comparator to observers' responses could produce the desired curves. ${ }^{4}$ While these modifications might be worth considering, we have chosen to take a more direct approach to modeling the data.

We leave unspecified the initial response of the visual system to the stimulus input (the impulse response) and details about the mechanisms responsible for arrival times at the hypothetical temporal order and simultane- 


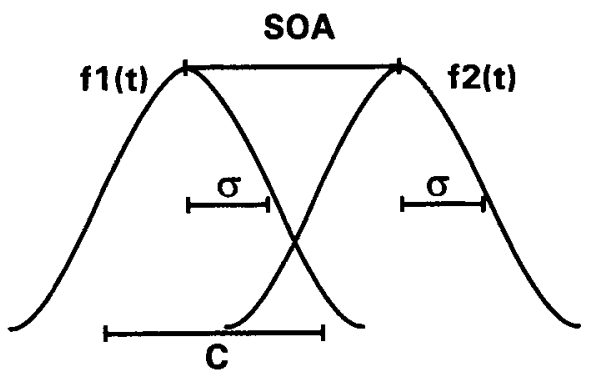

Figure 5. Illustration of the proposed model for judging the simultaneity of two stimuli. SOA is stimulus onset asynchrony; $f 1(t)$ and $f 2(t)$ are the probability densities for dating the two stimuli; $\sigma$ is the standard deviation of the uncertainty distributions, $f 1$ and $f 2$; $c$ is the critical interval for judging the simultaneity of the two stimuli.

ity comparators. We assume only that the date (absolute time) of each stimulus event is represented by a probability distribution. These distributions represent the uncertainty in dating the stimuli and are not identical to a temporal impulse response (in fact, they would be derived from the lower level mechanisms). We assume that if two stimuli are both dated within a certain critical interval, $c$, they are judged to be simultaneous. This is the same decision rule used by Jaśkowski's (1991) simultaneity comparator (although he derives the inputs to the simultaneity comparator from the transmission times of the stimulus signals). The probability that two stimuli with a given SOA are perceived as simultaneous is the probability that the stimulus dates fall within $c$ msec of each other. (Note that the calculations here are similar to those for a two-interval forced-choice signal-detection task.) The model is illustrated in Figure 5.

If the amount of attention allocated to the task is assumed to affect the size of the critical interval, $c$, with greater attention reducing the size of $c$, and if the uncertainties in dating the stimuli are represented by normal probability distributions, then the theoretical curves representing the probability that two stimuli are perceived as simultaneous, $P(\operatorname{sim})$, have the following properties: (1) $P(\operatorname{sim})$ is always less than one; (2) $P($ sim) decreases monotonically as $c$ decreases (as the allocation of attention increases); (3) $P(\operatorname{sim})$ is unimodal with a slope that is increasing near the center and then decreasing in the tails [note that $P(\operatorname{sim})$ itself is not a probability distribution]. The first two properties follow from the fact that the temporal uncertainty distributions are probability densities (the area under the curve is one) and the fact that $c$ is finite. The third property follows from the fact that the temporal uncertainty distributions are normal probability densities. Examples of the theoretical response curves produced when $c$ is varied are shown in Figure 6.

If the overall amount of attention allocated to a task is assumed to affect $c$, an attentional bias toward a location should also be reflected by a change in $c$. Such a bias is assumed to affect the symmetry of the critical interval, $c$. So, more generally, two events are perceived as simultaneous when $(-c / 2)+\epsilon<t_{2}-t_{1}<(c / 2)+\epsilon$, where $t_{1}$ and $t_{2}$ are the perceived dates of the two stimulus events. This is a straightforward modification of Jaśkowski's

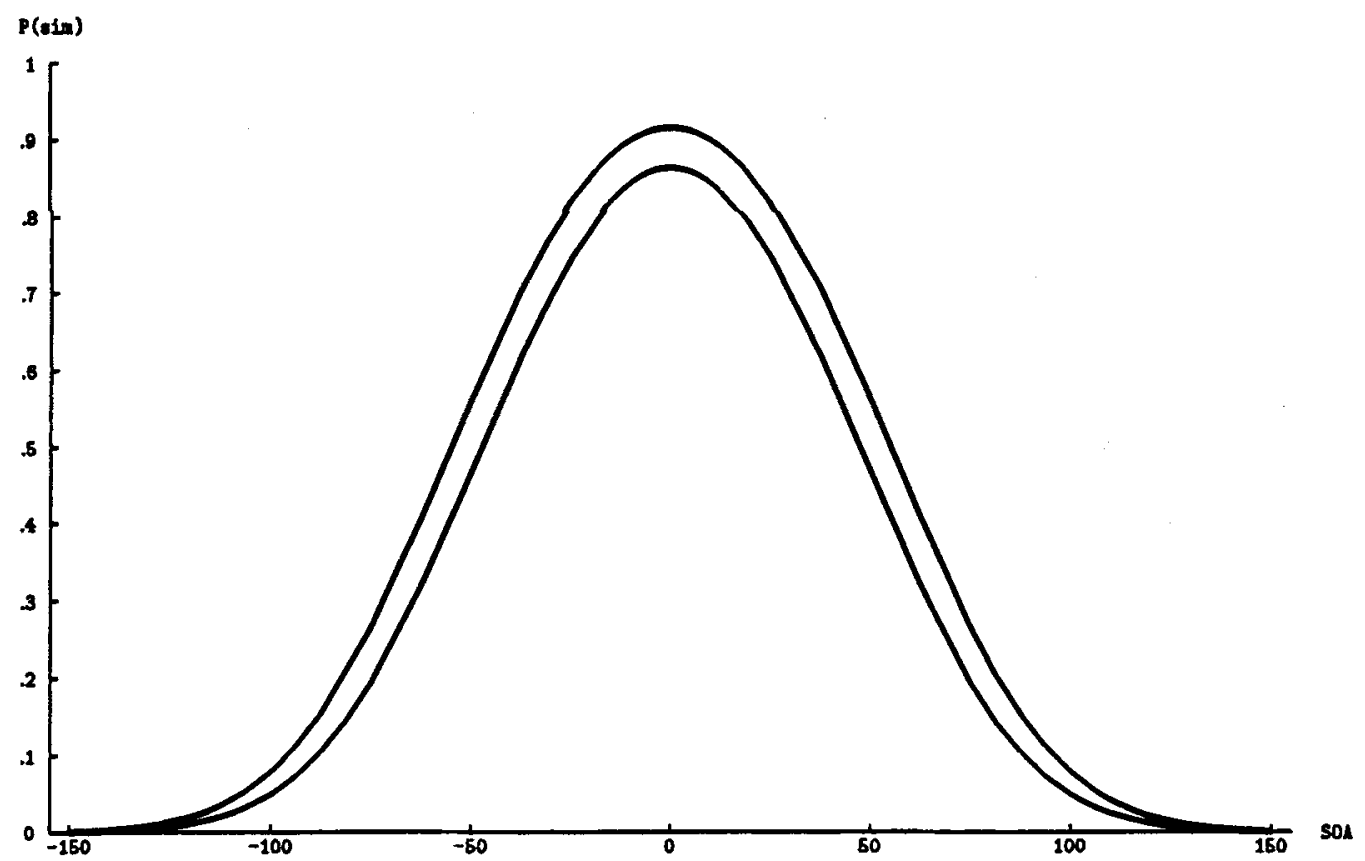

Figure 6. Predicted response curves from the model illustrated in Figure 5 when the critical interval $c$ is varied to represent changes in the overall allocation of attention to the task $(\sigma=22.5 ; c=110$, top curve; $c=95$, bottom curve). 


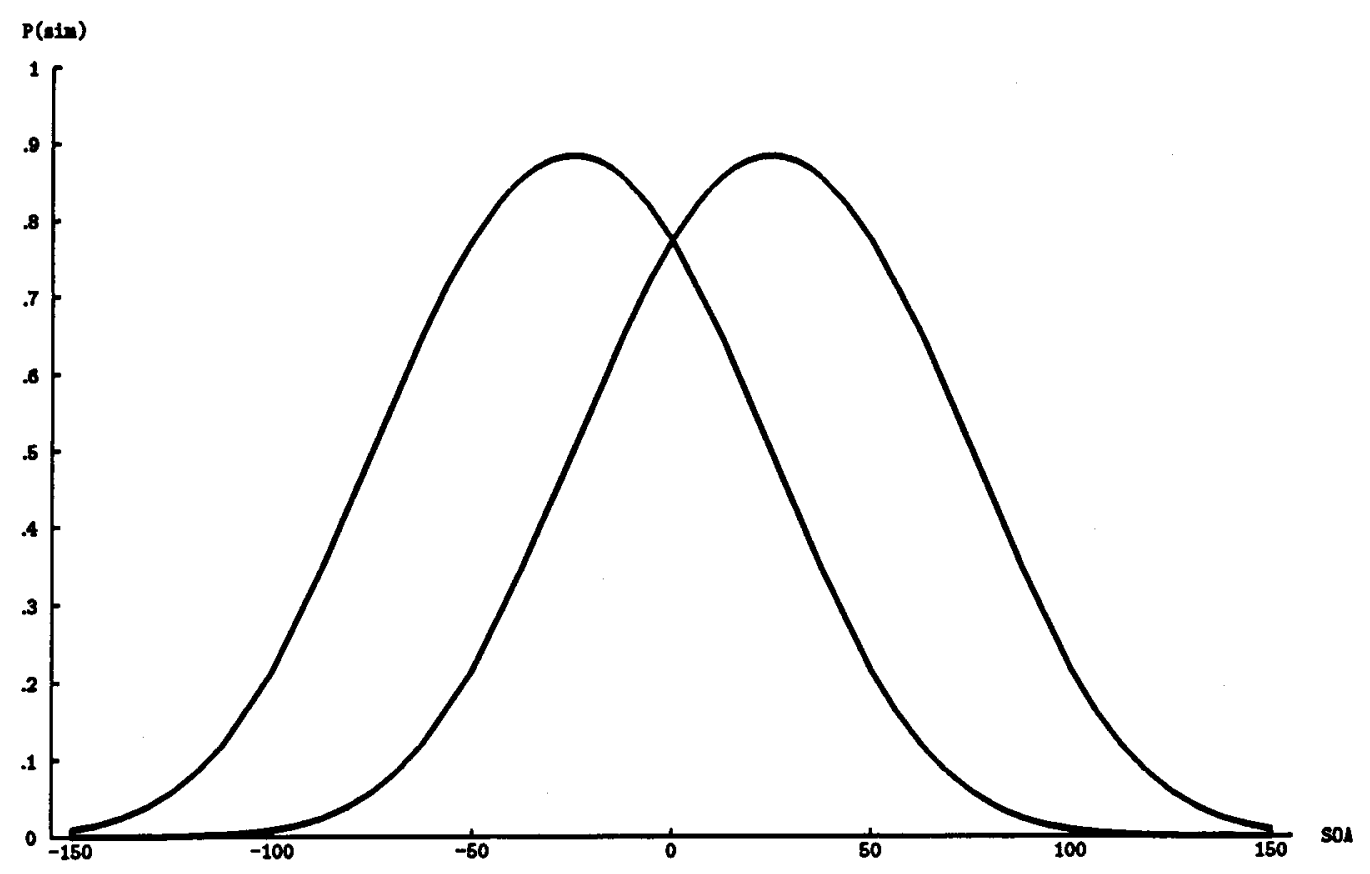

Figure 7. Predicted response curves from the model illustrated in Figure 5 when the location bias parameter $\epsilon$ is varied $(\sigma=22.5 ; c=100 ; \epsilon=-25$, left curve; $\epsilon=+25$, right curve).

(1991) decision rule presented in the introduction (Ulrich, 1987, also allows for an asymmetrical critical interval in the most general form of his model). The bias factor, $\epsilon$, reflects a shift of attention toward the location of one of the stimuli. The result is that the response distribution, $P(\operatorname{sim})$, is shifted away from the zero SOA position (see Figure 7). This is consistent with empirical findings. For example, Stelmach and Herdman (1991) found that the point of subjective simultaneity, the SOA at which two stimuli appear to be simultaneous, shifts about $20 \mathrm{msec}$ in favor of the onset of the less attended stimulus. This effect has recently been replicated by Carver and Brown (1997).

Methodologically, it is likely that the cues used to direct attention to a location also affect the amount of attention devoted to the task, in which case, in addition to the response curve being shifted, the shape of the curve, as determined by the size of the critical interval $c$, will change (cf. Figure 6). This occurs in Stelmach and Herdman (1991) and in Carver and Brown (1997), where the frequency of simultaneous responses decreases when attention is directed to only one of the stimulus locations.

Parameters of the model were estimated using Mathematica's FindMinimum routine (Wolfram, 1993). The 12 parameter estimates were found, which simultaneously minimized the goodness-of-fit measure $G^{2}$ for the four experimental conditions. $G^{2}$, a measure of fit based on the likelihood ratio of the obtained data given a model, has an asymptotic chi-square distribution. The parameter estimates for the data combined across observers are given in Table 1. The solid lines through the data points in Figures 3 and 4 represent the curves determined by the parameters shown in Table 1. As expected, the bias parameter $\epsilon$ and the uncertainty parameter $\sigma$ appear to remain relatively unchanged between conditions, whereas the size of the critical interval $c$, assumed to be affected by the allocation of attention, increases when less attention is devoted to the task. An analysis of variance of individual observers' parameter estimates showed the effect of cue validity on $c$ to be significant $[F(1,7)=10.8$, $p<.02]$. The effect of cue validity on $\epsilon$ was not significant $(F<1)$. However, the effect of cue validity on $\sigma$ was marginally significant $[F(1,7)=4.33, p=.08]$.

The pattern of results seen in Table 1 is maintained at the level of individual observers. Parameter estimates for individual observers are given in Table 2 . In this case, the parameters of the best fitting model are shown, meaning that parameters that do not significantly increase $G^{2}$ when held constant across the two cue-validity conditions were set equal across the cue-validity conditions. The model itself is a good fit for 7 of the 8 observers $(\mathrm{Ob}-$ servers $1,3,4,5,6,7,8$ ). The parameters vary signifi-

Table 1

Model Parameter Estimates for the Data Collapsed Across Observers

\begin{tabular}{lrrrrrrr}
\hline & \multicolumn{3}{c}{ Vertical Targets } & & \multicolumn{3}{c}{ Horizontal Targets } \\
\cline { 2 - 4 } \cline { 6 - 8 } & \multicolumn{1}{c}{$c$} & $\sigma$ & $\epsilon$ & & $c$ & $\sigma$ & $\epsilon$ \\
\hline Valid cue & 97.8 & 26.1 & 2.6 & & 120.9 & 24.5 & -4.7 \\
Invalid cue & 113.2 & 25.9 & 3.7 & & 130.9 & 22.1 & -5.9 \\
\hline
\end{tabular}

Note-The variable $c$ represents the critical interval for determining stimulus simultaneity; $\sigma$ represents the standard deviation of the temporal uncertainty distribution; and $\epsilon$ represents attentional location bias. As predicted, $c$ varies as a function of the overall allocation of attention to the task, whereas $\sigma$ and $\epsilon$ remain relatively constant. 
Table 2

Model Parameter Estimates for the Data for Individual Observers

\begin{tabular}{|c|c|c|c|c|c|c|c|}
\hline \multirow[b]{2}{*}{ Observer } & \multirow[b]{2}{*}{ Cue } & \multicolumn{3}{|c|}{ Vertical Targets } & \multicolumn{3}{|c|}{ Horizontal Targets } \\
\hline & & $c$ & $\sigma$ & $\epsilon$ & $c$ & $\sigma$ & $\epsilon$ \\
\hline \multirow[t]{2}{*}{1} & Valid & 77.2 & 23.8 & 8.4 & 136.4 & 21.5 & -1.5 \\
\hline & Invalid & 112.6 & 18.8 & 8.4 & 144.3 & 19.9 & -1 \\
\hline \multirow[t]{2}{*}{2} & Valid & 130.3 & 17.0 & 5.2 & 144.5 & 16.7 & -6.7 \\
\hline & Invalid & 133.0 & 17.0 & 5.2 & 166.3 & 16.7 & -6.7 \\
\hline \multirow[t]{2}{*}{3} & Valid & 97.5 & 19.4 & -3.0 & 114.6 & 16.5 & -5.6 \\
\hline & Invalid & 97.5 & 19.4 & -3.0 & 114.6 & 16.5 & -5.6 \\
\hline \multirow[t]{2}{*}{4} & Valid & 82.5 & 26.5 & 9.1 & 102.2 & 25.6 & -10.7 \\
\hline & Invalid & 109.2 & 25.0 & 9.1 & 111.9 & 21.2 & -10.7 \\
\hline \multirow[t]{2}{*}{5} & Valid & 97.0 & 14.1 & -3.7 & 97.6 & 14.0 & -3.0 \\
\hline & Invalid & 112.3 & 14.1 & -3.7 & 117.4 & 14.0 & -3.0 \\
\hline \multirow[t]{2}{*}{6} & Valid & 88.1 & 15.1 & -3.3 & 147.4 & 18.4 & -0.9 \\
\hline & Invalid & 88.1 & 15.1 & -3.3 & 147.4 & 18.4 & -0.9 \\
\hline \multirow[t]{2}{*}{7} & Valid & 46.5 & 22.7 & 0.7 & 63.3 & 19.0 & -3.3 \\
\hline & Invalid & 65.5 & 22.7 & 7.1 & 78.0 & 19.0 & -11.0 \\
\hline \multirow[t]{2}{*}{8} & Valid & 215.7 & 26.3 & 1.8 & 218.1 & 28.7 & -1.9 \\
\hline & Invalid & 262.8 & 28.2 & 13.7 & 213.0 & 21.7 & -8.8 \\
\hline
\end{tabular}

cantly as a function of cue validity for 6 of the 8 observers (Observers 1, 2, 4, 5, 7, 8).

It is clear from examining Table 2 that $c$ is the parameter most strongly affected by the allocation of attention. For the 6 observers whose performance was affected by cue validity, the parameter $c$ increases from the valid- to the invalid-cue conditions in 11 of 12 cases. We informally examined the influence of each of the model parameters on the overall fit of the model. The increase in $G^{2}$ when a parameter is held constant across cue validity provides an indication of the parameter's importance in determining the fit of the model; parameters producing a larger increase in $G^{2}$ when held constant have more influence on the fit of the model than do parameters producing a smaller increase. The parameter $c$ accounts for (by far) the largest change in $G^{2}$ of any of the parameters held constant across the cue-validity conditions: $\sigma$ produces a mean increase in $G^{2}$ of 6.2 when held constant across cue validity, $\epsilon$ produces a mean increase of 7.2, while $c$ produces a mean increase of 40.6 .

In other words, to the extent that the model captures the observers' performance, it appears that the attention manipulation had the greatest effect on the critical interval for determining simultaneity, and, based on the analysis of the combined data, possibly a small effect on the variability in determining the time of occurrence of the stimulus events. ${ }^{5}$

In sum, it would appear that the overall amount of attention allocated to making a judgment of the relative time of occurrence of two brief stimulus events affects an observer's ability to make that judgment, even when attention is distributed equally among the stimulus locations.

\section{REFERENCES}

ALLAN, L. G. (1975). The relationship between judgments of successiveness and judgments of order. Perception \& Psychophysics, 18, 29-36.

BORING, E. G. (1950). A history of experimental psychology. New York: Appleton-Century-Crofts.

CARVER, R., \& Brown, V. (1997). Combined effects of directed atten- tion and differential luminance on judgments of simultaneity. Manuscript submitted for publication.

Groner, M. T., BischoF, W. F., \& DiLollo, V. (1989). A model of visible persistence and temporal integration. Spatial Vision, 3, 293-304.

Hikosaka, O., MiYauCHI, S., \& SHIMOJO, S. (1993). Focal visual attention produces illusory temporal order and motion sensation. Vision Research, 33, 1219-1240.

JAMES, W. (1890). Principles of psychology. New York: Holt

JAŚKOWSKI, P. (1991). Two-stage model for order discrimination. Perception \& Psychophysics, 50, 76-82.

RouFs, J. A. (1963). Perception lag as a function of stimulus luminance Vision Research, 3, 81-91.

Roufs, J. A., \& BlommaerT, F. J. J. (1981). Temporal impulse and step responses of the human eye obtained psychophysically by means of a drift-correcting perturbation technique. Vision Research, 21, 1203-1221.

SekUler, R., Tynan, P., \& Levinson, E. (1973). Visual temporal order: A new illusion. Science, 180, 210-212.

Stelmach, L. B., \& Herdman, C. M. (1991). Directed attention and perception of temporal order. Journal of Experimental Psychology: Human Perception \& Performance, 17, 539-550.

StERnBERG, S., \& KNOLL, R. L. (1973). The perception of temporal order: Fundamental issues and a general model. In S. Kornblum (Ed.), Attention and performance IV (pp. 629-685). New York: Academic Press.

ULRICH, R. (1987). Threshold models of temporal-order judgments evaluated by a ternary response task. Perception \& Psychophysics, 42, 224-239.

WolfRAm, S. (1993). Mathematica: A system for doing mathematics by computer (Version 2.1) [Computer software]. Champaign, IL: Wolfram Research.

\section{NOTES}

1. The equation printed here is the general form of Equation 20 in Roufs and Blommaert (1981). Note that the analogous equation in Stelmach and Herdman (1991), Equation 2, contains a misprint.

2. Unfortunately, Stelmach and Herdman did not attempt to fit quantitative predictions of their model to their data. The model serves as a way to conceptualize the role of attention in their experimental paradigm. The model was used essentially as an analogy for how attentional processes might influence temporal order perception. In fact, in the form in which it was presented, the model could not be used to make quantitative predictions. Specifically, no rule was given for translating the output of the proposed temporal order/simultaneity comparators into response proportions; but it is necessary to state such a rule because the output of the simultaneity comparator, using the ratio of common-to-uncommon areas, is infinite at zero SOA. The model is, in fact, 
a hybrid from two initially unrelated sources: Roufs and Blommaert's (1981) paper on visual impulse responses and Groner, Bischof, and DiLollo's (1989) paper on visible persistence.

3. Note that only positive SOAs are plotted in the figure. The full response curve would inciude both positive and negative SOAs and would be symmetric about zero. Also, as the model is formulated, the output of the simultaneity comparator approaches infinity as SOA approaches zero. Thus, for purposes of illustration, we leave the values of the output of the hypothetical simultaneity comparator unspecified for SOAs near zero.

4. If Stelmach and Herdman's (1991) model is modified to include the response rule suggested by Groner, Bischof, and DiLollo (1989), the modified model and the model presented here should predict curves of similar shapes, since Groner et al.'s response rule is also based on integrating a Gaussian density function. Unfortunately, this response rule will not produce the desired difference in simultaneous responses as a function of attention at zero SOA, since the infinite ratio of commonto-uncommon areas will always map to a response proportion of 1 , regardless of the value of the parameter $b$. Also, while Stelmach and Herdman's model relies on largely unsubstantiated underlying mechanisms, the model presented here simply leaves the underlying mechanisms unspecified.

5. There is a shift in $\epsilon$ in the invalid-cue condition for Observers 7 and 8. This most likely reflects a natural attentional bias (cf. Sekuler, Tynan, \& Levinson, 1973) which is most readily apparent when less attention is devoted to the task. A similar increase in $\epsilon$ is seen as the luminance of the stimuli decreases (Carver \& Brown, 1997).

(Manuscript received July 3, 1995; revision accepted for publication June 12, 1996.) 